\title{
Acid microenvironments in microbial biofilms of antarctic endolithic microecosystems
}

\author{
Asunción de los Ríos, ${ }^{1}$ Jacek Wierzchos, ${ }^{2}$ \\ Leopoldo G. Sancho ${ }^{3}$ and Carmen Ascaso ${ }^{1 *}$ \\ ${ }^{1}$ Centro de Ciencias Medioambientales (CSIC) Serrano \\ 115 dpdo., Madrid-28006, Spain. \\ ${ }^{2}$ Servei de Microscopia Electrónica, Universitat de Lleida, \\ Rovira Roure 44, Lleida-25198, Spain. \\ ${ }^{3}$ Dpto. Biología Vegetal II, Universidad Complutense de \\ Madrid, Madrid-28040, Spain.
}

\section{Summary}

Antarctic endolithic microecosystems harbour distinct biofilms. The lithic substrate and the microorganisms comprising these films are intimately linked, leading to complex mineral-microbe interactions. Hence, the microhabitats and microenvironments of these microecosystems are not only determined by the physicochemical features of the lithic substrate, but are also conditioned by the biological components of these biofilms. The Antarctic biofilms analysed in this study are characterized by the presence of extracellular polymer substances and acid microenvironments in the proximity of the cells; cyanobacteria appearing as key components. On ultrastructural analysis, these endolithic cyanobacteria showed differences in sheath organization, probably related to their spatial position in the lithic substrate. It is proposed that in this type of ecosystem, biofilm structure could favour the formation of microsites with specific physicochemical conditions appropriate for the survival of microbial communities in this extreme environment.

\section{Introduction}

At the interface formed between the lithic substrate and atmosphere, accumulations of microorganisms are organized as biofilms (Little et al., 1997; Warscheid and Braams, 2000; De los Ríos et al., 2002). These biofilms are found at almost every interface, as long as it is exposed to water for at least a short time (Wimpenny et al., 2000). The biological components of a biofilm grow,

Received 5 December, 2002; accepted 5 December, 2002. *For correspondence. E-mail arios@ccma.csic.es; Tel. (+34) 917452500 ; Fax ( +34) 915640800 . reproduce and produce extracellular polymer substances (EPS), which frequently extend from the cell forming a tangled matrix of fibres as a structural assemblage (Characklis and Marshall, 1990). The organizational features of a biofilm are such that its microorganisms can maintain conditions at the biofilm-surface interface that are radically different from those of the bulk (Little et al., 1997).

Within the lithic substrate itself there are several types of microenvironment, the physicochemical conditions of which may differ according to the spatial distribution of the biofilm's components (Ascaso et al., 1998; de los Ríos et al., 2002). These microsites form, depending as much on the rock's physicochemical features as on the interactions among its different components. Factors such as $\mathrm{pH}$ can affect the formation of these microsites since they condition mineral alteration processes (Barker et al., 1998).

The set of microbial communities comprising the endolithic biofilm, their biotypes and inter-relationships constitute an ecosystem which, given its small size, is denoted a microecosystem. To characterize the endolithic microecosystem in detail, one must explore the living species and their habitats, along with the biogeochemical processes that take place in these microbial niches. Despite the vast amount of information on the effects of microorganisms on mineralogical processes and vice-versa in laboratory conditions, this complex interface has not been extensively explored in natural conditions. The reason for this is that it is extremely difficult to directly analyse this minute and dynamic space (Lower et al., 2001). The special features of the interface dictate the use of specific techniques including scanning electron microscopy (SEM) with backscattered electron (BSE) imaging (Wierzchos and Ascaso, 1994). This in situ investigation strategy was combined with an analytical X-ray energy dispersive spectroscopy (EDS) system, permitting the in situ study of the microorganism-lithic substrate interface and its interactions (Ascaso et al., 1995; Wierzchos and Ascaso, 1996; Lee and Parsons, 1999; de los Ríos et al., 2002; Wierzchos et al., 2003).

Of the existing extreme environments available for microbial diversity and ecology studies, Antarctic ecosystems are perhaps among the least described (Nichols et al., 1999). Much of life on the continent of Antarctica is 

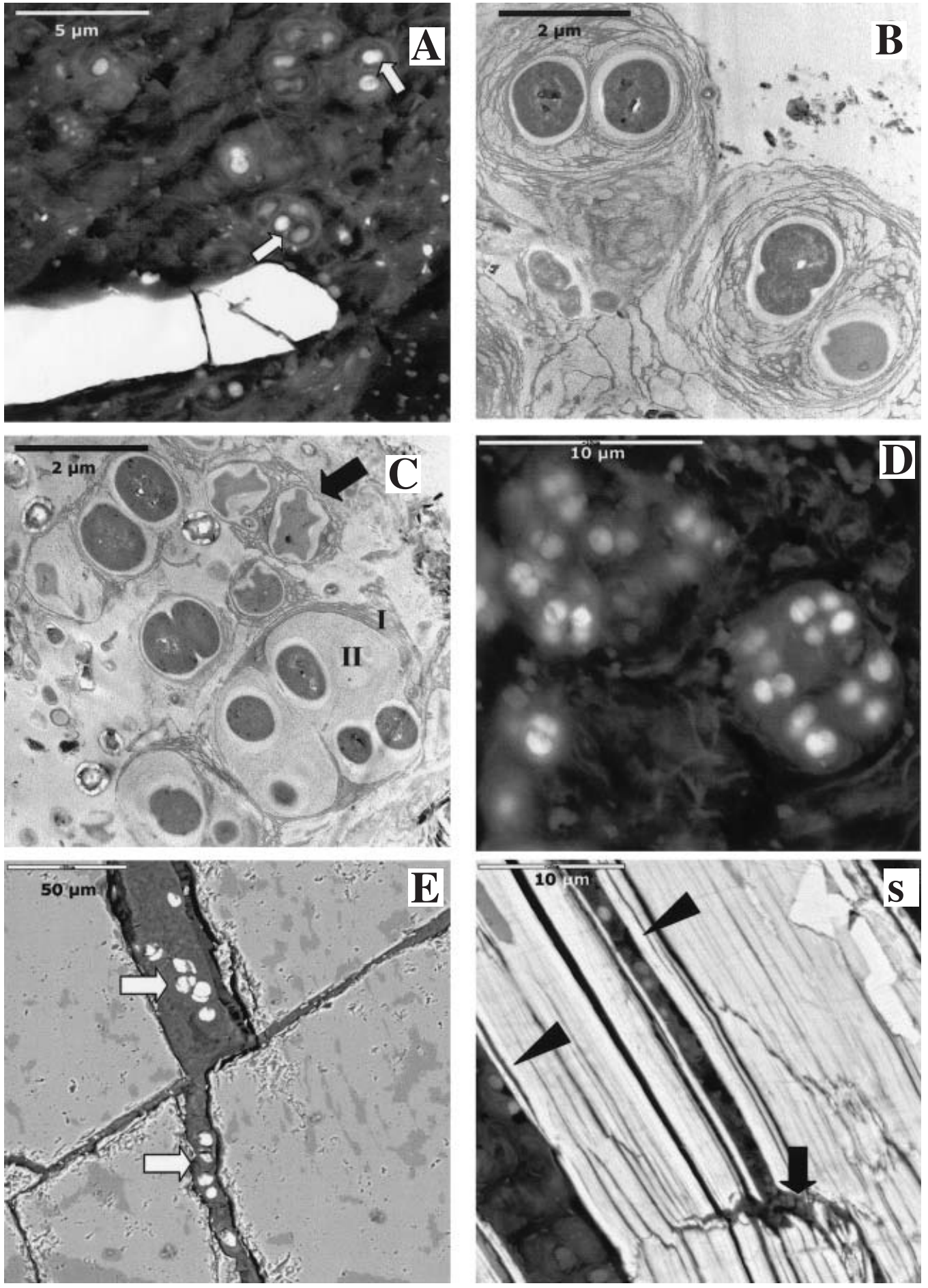

Fig. 1. A. SEM-BSE image of a cyanobacterial colony colonizing a near-surface fissure area.

B. TEM image showing cyanobacterial cells with sheaths comprising concentric electro-dense fibres.

C. TEM image showing cyanobacterial cells with sheaths comprised of two layers of different fibre arrangement (I and II). Arrow indicates collapsed dead cells.

D. SEM-BSE image of multicellular cyanobacterial aggregates in a deep fissure zone.

E. SEM-BSE image of a biofilm in which the fissure is mostly occupied by dead cells. Arrows indicate live cells.

F. SEM-BSE image of a cyanobacterial (arrowheads) and bacterial (arrow) colony observed between mica layers. 
restricted to endolithic microecosystems (Friedmann, 1982; Friedmann et al., 1988; Siebert et al., 1996; Banerjee et al., 2000; Wierzchos and Ascaso, 2001). The capacity of microorganisms to colonize internal zones of the rocks, means that microbial life exists in areas where the harsh environmental conditions prevent colonization of rock surfaces (Friedmann, 1982). If we are to understand how certain microorganisms are able to survive the extreme conditions of Antarctic rocks, besides their identification and physiological adaptations, the way in which integrating communities are organized within local microenvironments needs to be established. Hence, the aim of this study was to characterize several of the microenvironments of the lithic microecosystems of Antarctica by combining the microscopy techniques: confocal laser scanning microscopy (CSLM), SEM-BSE and transmission electron microscopy (TEM). Special attention was paid to establishing $\mathrm{pH}$ conditions and observing the ultrastructure of the microorganisms in each zone.

\section{Results}

The fissures and cavities of Antarctic rocks from the Granite Harbour zone are occupied by endolithic microbial communities (Fig. 1A). In zones where there were no lichen thalli close by, cyanobacterial colonies were the main components of the communities observed. The ultrastructure of these cyanobacteria was explored by TEM. Multicellular aggregates were observed, each being enveloped in a common sheath. Fibres running parallel to the cell surface were the main components of the sheath of some aggregates (Fig. 1B). However, in another groups of cells, two different layers could be distinguished in the sheath: an outer layer of concentric agglutinations of fibres (I) and an inner, less electro-dense layer with thinner and more irregularly distributed fibres (II) (Fig. 1C). Dead cells appeared highly collapsed, but an envelope of concentric electro-dense fibres was still visible (arrow in Fig. 1C). According to their morphology and ultrastructure, most of these cyanobacterial cells were identified as belonging to the Gloeocapsa group. Differences in the cyanobacterial sheath could also be discerned by SEMBSE. Some cells showed a sheath with an internal halo emitting a lower BSE signal (arrows in Fig. 1A). These cells, which were found mainly in fissures close to the surface, could correspond to the second type observed by TEM in which two different sheath layers were distinguished. However, other cells showed a more filamentous and homogeneous sheath (Fig. 1D). These cells were located deeper in the fissures and probably corresponded to the first type described by TEM. Biofilms with a few live cells were observed in some deep fissures. In these cases, the entire fissure was occupied by sheath and cell remnants mixed with these live cells (Fig. 1E).
Lithobiontic communities are intimately related to the minerals of the lithic substrate. Figure 1F shows the presence of cyanobacteria (arrowheads) and bacteria (arrow) among micaceous layers in the process of exfoliation. Figure 2A shows spatial distribution maps of the elements silicon, aluminium and potassium in an area of these exfoliated micas. These maps were provided by the EDS microanalytical system. The arrows show a zone of potassium depletion in the proximity of cyanobacterial cells.

By examining Lucifer yellow-stained samples by confocal microscopy, these microbial communities can be seen to be embedded in a polymeric matrix. Figure $2 \mathrm{~B}$ shows cyanobacteria and bacteria (arrow) and filamentous fungi (arrowhead) within in a polymeric matrix inside an Antarctic rock fissure. The use of three-dimensional colour spectacles gives information on the spatial organization of these biofilm components. The confocal micrograph in Fig. $2 \mathrm{C}$ shows a deep-coded three-dimensional image of an Antarctic biofilm composed of cyanobacteria. This image shows the spatial distribution of these microorganisms in the lithic substrate and also allows estimation of the number of microorganisms present in the fissure. Cyanobacteria were distributed over the rock surface but were also seen to inhabit many endolithic fissures at a density estimated at $10^{5}$ cells per $\mathrm{mm}^{3}$ of fissure.

The $\mathrm{pH}$ determinations made by confocal microscopy and Cl-NERF staining indicated distinctly acidic environments in the proximity of endolithic communities. Figure 2D shows the $\mathrm{pH}$ range recorded in the area surrounding a colony of cyanobacteria inside a lithic cavity. Values of $\mathrm{pH}$ lower than 3.5 were registered close to the cells.

\section{Discussion}

The Antarctic microecosystems analysed in this study are organized as biofilms in which cyanobacteria appear as a major component. These films can be viewed as systems embedded in an organic matrix resulting from the excretion of polymers by resident microorganisms. As a result of the interplay among their components, these systems are highly dynamic. Interrelations do not only involve the biological components but also the immediate environment in which they are found. This biocomplexity was examined in detail by combining several in situ microscopy techniques.

The major presence of cyanobacteria in these biofilms was not unexpected since they are especially prevalent in harsh, arid conditions (García-Pichel and Belnap, 1996; García-Pichel et al., 2001). As shown by SEM-BSE, some of the features of the cyanobacterial sheaths could be correlated with their position in the rock. Variations in the cyanobacterial envelope may reflect different physiological stages or levels of adaptation to the environment. Grilli Caiola et al. (1993) suggested that an increased content 

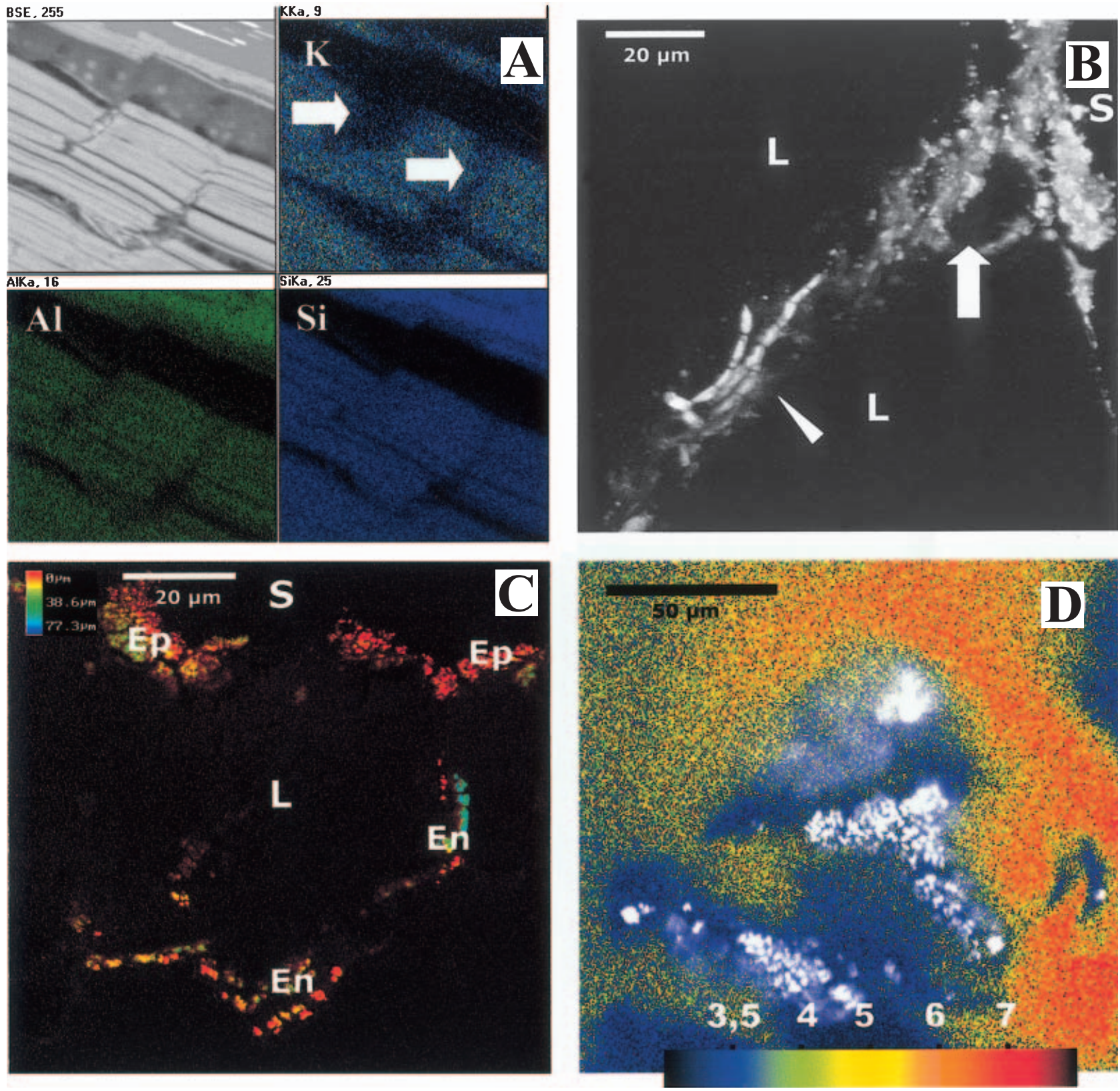

Fig. 2. A. EDS spatial distribution of potassium (K), aluminium (Al) and silicon (Si) in exfoliated mica layers. Arrows indicate zones of potassium depletion.

B. Three dimensional reconstruction of CSLM sections through a Lucifer yellow-stained specimen showing a microbial community embedded in a polymeric matrix in relation to the rock surface. The stereoimage can be observed using three-dimensional colour spectacles. The arrow points to a bacterial and cyanobacterial aggregate. Arrowhead indicates fungal cells. L: lithic substrate; S: surface.

C. CSLM deep-coded image of cyanobacterial cells occupying epilithic and endolithic positions. L: lithic substrate; S: surface; Ep epilithic cyanobacteria; En: endolithic cyanobacteria.

D. CSLM images of a specimen stained with Cl-NERF (cell-impermeant $\mathrm{pH}$-sensitive dye) showing the pH in a cavity occupied by a colony of cyanobacteria. This image was compiled by combining the image obtained by excitation with a 488-nm laser and a BP $515 \mathrm{~nm}$ emission filter with that produced by a 543-nm excitation laser and LP $570 \mathrm{~nm}$ emission filter.

of hydrophobic compounds in the envelope could reduce water loss but also limit exchange with the environment. These authors reported that cells of the cyanobacterium Chroococcidiopsis with multilayered or thick envelopes had an intact cytoplasm structure, whereas altered cell structures were observed in cells with firm electro-dense envelopes. We could refer to similar phenomena here, because dead cells showed thick, dense sheaths. 
The presence of cyanobacteria enclosed in a polymeric matrix in zones of mica exfoliation and potassium biomobilization processes suggests their contribution to such processes. These phenomena have also been observed under lichen thalli and were associated with acid production and the presence of polymers (Wierzchos and Ascaso, 1996; 1998; de los Ríos et al., 2002). Further, the contribution of saxicolous lichens to biochemical weathering in relation to the influx of elements from the rock has been recently quantitatively demonstrated (Aghamiri and Schwartzman, 2002). The intimate relationship between the lithic substrate and microorganisms gave rise to complex mineral-microbe interactions such as those observed here. Microorganisms affect mineralogical processes and vice-versa (see Lower et al., 2001). One of the characteristics shared by all the forms of life represented by the term biofilm is that the microorganisms are embedded in a matrix of extracellular polymeric substances (EPS, Wingender et al., 1999). The use of microscopy techniques allowed us to detect these EPS in situ in biofilms found in Antarctic rocks. Microbial-enclosing polymer layers can suppress or enhance chemical weathering by up to three orders of magnitude, depending on the $\mathrm{pH}$, mineral structure and composition, and organic groups (Barker et al., 1998; Banfield et al., 1999). There is a strong dependence of most mineral dissolution rates on pH (Barker et al., 1998; Welch et al., 2002) and it was confirmed that the presence and activity of these cyanobacteria gives rise to $\mathrm{pH}$ gradients in the fissures and cavities of the substrate. The low $\mathrm{pH}$ zones detected were always in close proximity to the microorganisms and most likely favoured the geochemical alteration processes observed. Similar acidic environments were detected around bacterial cells found within biotite cleavages in laboratory studies by Barker et al. (1998). The presence of acidic extracellular polymers together with the production of inorganic and organic acids could account for the acid $\mathrm{pH}$ registered. Microorganisms within these biofilms seem to be capable of sustaining environments different from those of the bulk in terms of $\mathrm{pH}$ among other factors. The low $\mathrm{pH}$ of these microenvironments could affect the development of the communities comprising the biofilm in two ways. On the one hand, $\mathrm{pH}$ values can affect bioweathering and thus microhabitat formation and, on the other, only microorganisms able to tolerate such low $\mathrm{pH}$ conditions could survive in these biofilms.

The occurrence and abundance of organisms in an environment are determined by factors that must remain within the tolerance range of the organism. The role of purely physicochemical factors in regulating biofilm structure needs to be considered in tandem with genotypic and phenotypic factors (Wimpenny et al., 2000). In these endolithic biofilms, different microhabitats and microenvironments are produced, which, in turn, are probably col- onized by a number of microorganisms that favour or are favoured by these specific conditions. The final result of this type of organization is that zones are created, in which the physical and chemical conditions may vary such that they are often much less severe than those of the external environment. The biofilm strategy adopted by these Antarctic microorganisms may indeed protect them from the harsh environment (Flemming, 1993; Davey and O'Toole, 2000). Thus, safe within the biofilm structure, microbial survival in endolithic Antarctic microenvironments may be more related to avoiding than being able to tolerate extreme conditions outside the microhabitat.

\section{Experimental procedures}

\section{Materials}

Pieces of granite rock with scarce colonization by lichens and mosses were collected from the Ross Sea coast, Granite Harbour $\left(77^{\circ} 00^{\prime} \mathrm{S}, 162^{\circ} 34^{\prime} \mathrm{E}\right)$. In this zone, epilithic forms of life were only present in places regularly exposed to melt water (Seppelt et al., 1995). Samples were collected under natural conditions and stored at $-20^{\circ} \mathrm{C}$ until processing for microscopy and microanalytical procedures.

\section{SEM-BSE, EDS and CSLM}

The samples were prepared according to a procedure developed for observing the rock-microorganism interface by SEM-BSE (Wierzchos and Ascaso, 1994). In brief, the pieces of rock were first fixed in glutaraldehyde and then in osmium tetroxide (only for SEM-BSE), dehydrated in a series of ethanol solutions and embedded in LR-White resin. Blocks of resin-embedded rock samples were finely polished, carbon coated (only for SEM-BSE) and observed using DMS 960 and DMS 940 A Zeiss SEM microscopes and an LSM 310 Zeiss confocal microscope. Microprobe analyses were performed using an energy dispersive spectrometry (EDS) Link ISIS microanalytical system during SEM observation.

\section{TEM}

Cyanobacteria were removed from the rock under the stereo microscope using a sterile needle and blocked in $2 \%(\mathrm{w} / \mathrm{v})$ agar. Small pieces of agar containing the cyanobacterial cells were fixed in glutaraldehyde and osmium tetroxide solutions, dehydrated in a graded ethanol series, and embedded in Spurr's resin following the protocol described in De los Ríos and Ascaso (2001). Ultrathin sections were poststained with lead citrate (Reynolds, 1963) and observed in a Zeiss EM910 transmission electron microscope.

\section{Extracellular $\mathrm{pH}$ determinations}

The $\mathrm{pH}$ measurements were made using Cl-NERF (cellimpermeant, $\mathrm{pH}$-sensitive dye for use in acidic environments, Molecular Probes) according to the procedure described by Barker et al. (1998). Fluorescence emission spectra were 
obtained using an LSM 310-Zeiss confocal microscope. Standard curves were prepared with the dye solution titrated to $\mathrm{pH}$ values in the range $2.5-7$, followed by confocal microscope imaging.

\section{Acknowledgements}

The authors would like to thank Fernando Pinto for technical assistance, Ana Burton for reviewing the English and Antarctic New Zealand for their logistic support and excellent field facilities. Financial support was provided by the DGICYT projects ANT99-0680 and BOS2000-1121.

\section{References}

Aghamiri, R., and Schwartzman, D.W. (2002) Weathering rates of bedrock by lichens: a mini watershed study. Chem Geol 188: 249-259.

Ascaso, C., Wierzchos, J., and de los Ríos, A. (1995) Cytological investigations of lithobiontic microorganisms in granitic rocks. Bot Acta 108: 474-481.

Ascaso, C., Wierzchos, J., and de los Ríos, A. (1998) In situ cellular and enzymatic investigations of saxicolous lichens using correlative microscopical and microanalytical techniques. Symbiosis 24: 221-234.

Banerjee, M., Whitton, B.A., and Wynn-Williams, D.D. (2000) Phosphatase activities of endolithic communities in rocks of the Antarctic Dry Valleys. Microbial Ecol 39: 8091.

Banfield, J.F., Barker, W.W., Welch, S.A., and Taunton, A. (1999) Biological impact on mineral dissolution: application of the lichen model to understand mineral weathering in the rizosphere. Proc Natl Acad Sci USA 96: 34043411.

Barker, W.W., Welch, S.A., Chu, S., and Banfield, J.F. (1998) Experimental observations of the effects of bacteria on aluminiosilicate weathering. Am Mineralogist 83: 15511563.

Characklis, W.G., and Marshall, K.C. (1990) Biofilms: a basis for an interdisciplinary approach. In Biofilms. Characklis, W.G., and Marshall, K.C. (eds). New York: John Wiley \& Sons, pp. 4.

Davey, M.E., and O'Toole, G.A. (2000) Microbial biofilms: from ecology to molecular genetics. Microbiol Mol Biol Rev 64: 847-867.

Flemming, H.C. (1993) Biofilms and environmental protection. Water Sci Technol 27: 1-10.

Friedmann, E.I. (1982) Endolithic microorganisms in the Antarctic Cold Desert. Science 215: 1045-1053.

Friedmann, E.I., Hua, M., and Ocampo-Friedmann, R. (1988) Cryptoendolithic lichen and cyanobacterial communities of the Ross Desert, Antarctica. Polarforschung 58: 251259.

García-Pichel, F., and Belnap, J. (1996) Microenvironments and microscale productivity of cyanobacterial desert crusts. J Phycol 32: 774-782.

García-Pichel, F., López-Cortés, A., and Nübel, U. (2001) Phylogenetic and morphological diversity of cyanobacteria in soil desert crusts from the Colorado Plateau. Appl Environ Microbiol 67: 1902-1910.
Grilli Caiola, M., Ocampo-Friedmann, R., and Friedmann, E.I. (1993) Cytology of long-tern desiccation in the desert cyanobacterium Chroococcidiopsis (Chroococcales). Phycologia 32: 315-322.

Lee, M.R., and Parsons, I. (1999) Biomechanical and biochemical weathering of lichen-encrusted granite: textural controls on organic-mineral interactions and deposition of silica-rich layers. Chem Ecol 161: 385-397.

Little, B.J., Wagner, P.A., and Lewandowski, Z. (1997) Spatial relationships between bacteria and mineral surfaces. In Geomicrobiology: Interactions Between Microbes and Minerals. Banfield, J., and Nealson, K.H. (eds). Washington D.C.: Mineral Society of America, pp. 123-189.

Lower, S.K., Tadanier, C.J., and Hochella, M.F.J. (2001) Dynamics of the mineral-microbe interface: use of biological force microscopy in biogeochemistry and geomicrobiology. Geomicrobiol J 18: 63-76.

Nichols, D., Bowman, J., Sanderson, K., Mancuso Nichols, C., Lewis, T., McMeekin, T., and Nichols, P.D. (1999) Developments with Antarctic microorganisms: culture collections, bioactivity screening, taxonomy, PUFA production and cold adapted enzymes. Curr Opinion Biotechnol 10: 240-246.

Reynolds, S. (1963) The use of lead citrate at high $\mathrm{pH}$ as an electron-opaque stain in electron microscopy. J Cell Biol 17: 200-211.

de los Ríos, A., and Ascaso, C. (2001) Preparative techniques for transmission electron microscopy and confocal laser scanning microscopy of lichens. In Protocols in Lichenology. Kranner, I., Beckett, R.P., and Varma, A.K. (eds). Berlin: Springer, pp. 85-117.

de los Ríos, A., Wierzchos, J., and Ascaso, C. (2002) Microhabitats and chemical microenvironments under saxicolous lichens growing on granite. Microb Ecol. 43: 181-188.

Seppelt, R.D., Green, T.G.A., and Schroeter, B. (1995) Lichens and mosses from the Kar Plateau, Southern Victoria Land, Antarctica. NZ J Bot 33: 203-220.

Siebert, J., Hirsch, P., Hoffmann, B., and Gliesche, C.G. (1996) Cryptoendolithic microorganisms from Antarctic sandstone of Linnaeus Terrace (Asgard Range): diversity, properties and interactions. Biodiversity Conservation 5: 1337-1363.

Warscheid, T., and Braams, J. (2000) Biodeterioration of stone: a review. Int Biodeterioration Biodegradation 46: 343-368.

Welch, S.A., Taunton, A.E., and Banfield, J.F. (2002) Effect of microorganisms and microbial metabolites on apatite dissolution. Geomicrobiol J 19: 343-367.

Wierzchos, J., and Ascaso, C. (1994) Application of BackScattered electron imaging to the study of the lichen rock interface. J Microscopy-Oxford 175: 54-59.

Wierzchos, J., and Ascaso, C. (1996) Morphological and chemical features of bioweathered granitic biotite induced by lichen activity. Clays Clay Minerals 44: 652-657.

Wierzchos, J., and Ascaso, C. (1998) Mineralogical transformation of bioweathered granitic biotite, studied by HRTEM: evidence for a new pathway in lichen activity. Clays Clay Minerals 46: 446-452.

Wierzchos, J., and Ascaso, C. (2001) Life, decay and fossilisation of endolithic microorganisms from the Ross Desert, Antarctica. Polar Biol 24: 863-868. 
Wierzchos, J., Ascaso, C., Sancho, L.G., and Green, A. (2003) Iron-rich diagenetic minerals are biomarkers of microbial activity in Antarctic rocks. Geomicrobiol $J$ (in press).

Wimpenny, J., Manz, W., and Szeewzyk, U. (2000) Heterogeneity in biofilms. FEMS Microbiol Rev 24: 661-671.
Wingender, J., Neu, T.R., and Flemming, H.C. (1999) What are bacterial extracellular polymeric substances? In Microbial Extracellular Polymeric Substances. Wingender, J., Neu, T.R., and Flemming, H.C. (eds). Berlin: Springer, pp. $1-19$. 